\title{
Video Article \\ Depletion of Mouse Cells from Human Tumor Xenografts Significantly Improves Downstream Analysis of Target Cells
}

\author{
David J. Agorku ${ }^{1}$, Stefan Tomiuk ${ }^{1}$, Kerstin Klingner ${ }^{2}$, Stefan Wild ${ }^{1}$, Silvia Rüberg ${ }^{1}$, Lisa Zatrieb ${ }^{1}$, Andreas Bosio ${ }^{1}$, Julia Schueler ${ }^{2}$, Olaf Hardt ${ }^{1}$ \\ ${ }^{1}$ R\&D Reagents, Miltenyi Biotec $\mathrm{GmbH}$ \\ ${ }^{2}$ In vivo Tumorbiology, Oncotest $\mathrm{GmbH}$
}

Correspondence to: David J. Agorku at davida@miltenyibiotec.de

URL: https://www.jove.com/video/54259

DOI: doi:10.3791/54259

Keywords: Medicine, Issue 113, oncology, cancer stem cells, tumor xenograft models, xenografted tissues, drug development, preclinical modelling, cell line establishment, Next Generation Sequencing, whole exome sequencing, tumor cell culture, SNP calling, cancer biology

Date Published: 7/29/2016

Citation: Agorku, D.J., Tomiuk, S., Klingner, K., Wild, S., Rüberg, S., Zatrieb, L., Bosio, A., Schueler, J., Hardt, O. Depletion of Mouse Cells from Human Tumor Xenografts Significantly Improves Downstream Analysis of Target Cells. J. Vis. Exp. (113), e54259, doi:10.3791/54259 (2016).

\section{Abstract}

The use of in vitro cell line models for cancer research has been a useful tool. However, it has been shown that these models fail to reliably mimic patient tumors in different assays ${ }^{1}$. Human tumor xenografts represent the gold standard with respect to tumor biology, drug discovery, and metastasis research ${ }^{2-4}$. Tumor xenografts can be derived from different types of material like tumor cell lines, tumor tissue from primary patient tumors ${ }^{4}$ or serially transplanted tumors. When propagated in vivo, xenografted tissue is infiltrated and vascularized by cells of mouse origin. Multiple factors such as the tumor entity, the origin of xenografted material, growth rate and region of transplantation influence the composition and the amount of mouse cells present in tumor xenografts. However, even when these factors are kept constant, the degree of mouse cell contamination is highly variable.

Contaminating mouse cells significantly impair downstream analyses of human tumor xenografts. As mouse fibroblasts show high plating efficacies and proliferation rates, they tend to overgrow cultures of human tumor cells, especially slowly proliferating subpopulations. Mouse cell derived DNA, mRNA, and protein components can bias downstream gene expression analysis, next-generation sequencing, as well as proteome analysis ${ }^{5}$. To overcome these limitations, we have developed a fast and easy method to isolate untouched human tumor cells from xenografted tumor tissue. This procedure is based on the comprehensive depletion of cells of mouse origin by combining automated tissue dissociation with the benchtop tissue dissociator and magnetic cell sorting. Here, we demonstrate that human target cells can be can be obtained with purities higher than $96 \%$ within less than 20 min independent of the tumor type.

\section{Video Link}

The video component of this article can be found at https://www.jove.com/video/54259/

\section{Introduction}

Solid human tumors consist of multiple physiological as well as neoplastic cell types. They form heterogeneous tissues with complex biological structures. Biological processes like tumor formation, progression and responses towards therapies are not yet fully understood. In vitro cell culture models represent a useful tool to study and understand tumor biology. However, they can only partly mirror structures and processes found in tumor tissues. Reliable and stable preclinical human tumor models are a prerequisite for the development of anti-tumor drugs and therapies ${ }^{4,6}$ as well as for understanding tumor biology and the interaction of tumor cells and their environment.

Human tumor xenograft models derived from primary patient tumors show high relations to the tissue of origin regarding histological architecture, interactions with micro-environmental structures, metastatic potential and responses to drugs ${ }^{7}$. Even when tumor xenografts are derived from cultured cells or cell lines they more closely mimic patient tumors, therefore showing higher validity in most assays compared to in vitro cell culture models. These features make them the gold standard of preclinical models ${ }^{4}$. Besides its application in cancer research, xenotransplantation of human cells into mice is also frequently used in stem cell research to determine the stemness and differentiation potential of a target population.

It has been shown that human microvasculature and human immune cells are replaced upon in vivo propagation of human tumors ${ }^{2,9}$. Factors such as the tumor subtype, growth rate, and region of transplantation have major impacts on the global level of infiltration as well as the composition of the mouse cell types found. However, even when these factors are kept constant, the amount and composition of mouse cells are highly variable.

Downstream analyses of xenografted tissues are often challenged by cells of murine origin. Primary cultures of human tumor cells from xenografts are frequently overgrown by fibroblasts. Besides hampering the generation of tumor cell lines in vitro, downstream assays such as drug cytotoxicity testing or pharmacokinetics are biased since in silico correction for effects originating from contaminating mouse cells is impossible in most cases. Beyond that, the only partly elucidated cross-talk between mouse fibroblasts and human tumor cells has a direct 
impact on experimental outcomes of studies ${ }^{10}$. Furthermore, the most widely unpredictable variation of infiltrating mouse cells aggravates accurate molecular downstream analyses. In NGS or proteome analyses each mouse-derived signal measured instead of a human tumor signal directly decreases sequencing sensitivity. Also microarray based expression analyses are challenged by murine nucleic acids possibly cross hybridizing to human probes.

In order to circumvent the obstacles of contaminating mouse cells in downstream analyses of human tumor cells from xenograft models different approaches have been proposed. In many studies desired target cells for downstream analysis are isolated by utilizing markers or combinations of markers exclusively expressed on human tumor cells. However, the lack of reliable markers for positive identification of human tumor cells frequently represents a big hurdle. Even broadly expressed markers, such as EpCAM on human carcinomas, frequently show tumor intrinsic expression differences ${ }^{11}$. This increases the risk that low expressing subpopulations, e.g., tumor cells undergone epithelial-to-mesenchymal transition, are lost during isolation. In addition, the direct binding of a selection agent to the target cell might influence the subsequent analysis results. Attempts of depleting mouse cells by using a combination of antibodies specific for murine CD45 and MHC class I epitopes have also been made ${ }^{12}$. However, this marker combination only detects a subset of mouse cells. Another approach is to enhance analyzing processes by software. Nevertheless, all these approaches are either not suitable for any kind of experimental setup or for any tumor entity and transplantation method.

In this study we present a novel and fast method for comprehensively depleting mouse cells from xenografted tissues independent of the mouse strain and tissue of origin. In screenings on multiple target organs and tissues for transplantation of xenografts (including skin, lung, brain, kidney and skeletal muscle) we were able to identify a combination of antibodies allowing for comprehensively detecting mouse cells. These antibodies were coupled to superparamagnetic particles, titrated and optimized for depletion by using magnetic cell sorting. As only mouse specific antibodies are used, human target cells stay "untouched" and the method is not limited to xenotransplanted tumor tissue. We demonstrate that comprehensively removing mouse cells from xenografted tumor samples standardizes and facilitates cultures of human tumor cells. Furthermore, we show that the analysis of human tumor xenografts by next generation sequencing is significantly improved. As this effect was observed although a human sequence specific selection has been carried out during exome enrichment, the influence on whole genome and whole transcriptome sequencing are expected to be even more prominent. Taken together, removal of mouse cells using this novel method facilitates cultivation of human tumor cells and significantly improves the downstream analyses of human tumor xenografts.

\section{Protocol}

All animal experiments were performed in accordance with local ethical and legal guidelines of Regierungspräsidium Freiburg Referat 35.

\section{Reagent Preparation}

NOTE: Before starting the experiment, prepare the following reagents from the dissociation kit:

1. To prepare enzyme $\mathrm{H}$ dissolve each vial of the lyophilized powder in $3 \mathrm{ml}$ of RPMl 1640 Roswell Park Memorial Institute 1640 (RPMI 1640) or Dulbecco's modified Eagle medium (DMEM). In order to avoid repeated freezing and thawing prepare suitable aliquots (e.g., $200 \mu \mathrm{l})$ and store them at $-20^{\circ} \mathrm{C}$ for up to 6 months.

2. To prepare enzyme R dissolve the vial of the lyophilized powder in $2.7 \mathrm{ml}$ of RPMI or DMEM medium. In order to avoid repeated freezing and thawing prepare suitable aliquots (e.g., $100 \mu \mathrm{l})$ and store them at $-20^{\circ} \mathrm{C}$ for up to 6 months.

3. To prepare enzyme A dissolve the vial of the lyophilized powder in $1 \mathrm{ml}$ of Buffer A supplied with the kit without vortexing. In order to avoid repeated freezing and thawing prepare suitable aliquots (e.g., $25 \mu \mathrm{l})$ and store them at $-20^{\circ} \mathrm{C}$ for up to 6 months. Make sure to thoroughly mix this suspension immediately before withdrawing the required reaction volume.

4. Prepare $250 \mathrm{ml}$ buffer (1x PBS with $0.5 \% \mathrm{BSA}$ ) for cell separation procedure and antibody staining NOTE: When culturing cells after removal of mouse cells filter all buffers and enzyme solutions through a $0.22 \mu \mathrm{m}$ filter.

\section{Tumor Dissociation Protocol}

NOTE: In this study patient-derived xenografts of non-small cell lung cancer (NSCLC), renal cancer and bladder were used. The tumors were generated by implanting xenografts of non-small cell lung cancer (NSCLC) renal cancer and bladder in 4 - 6 weeks old female NMRI nu/nu mice as described previously ${ }^{13}$.

NOTE: As this protocol is completely independent of the tumor type, it can be used for any kind of patient or cell line-derived tumor as well as other types of human xenotransplanted tissue without modification of the protocol.

1. Prepare $5 \mathrm{ml}$ of digestion mix per tissue sample (up to $1 \mathrm{~g}$ ) freshly by adding $4.7 \mathrm{ml}$ of RPMl 1640 or DMEM, $200 \mu \mathrm{l}$ of enzyme $\mathrm{H}, 100 \mu \mathrm{l}$ of enzyme R, and $25 \mu \mathrm{l}$ of enzyme A into a cellular dissociation tube (C Tube). Make sure to thoroughly mix enzyme $\mathrm{R}$ suspension before withdrawing the required volume.

2. Sacrifice the animals by cervical dislocation under anesthesia. Disinfect mouse by spraying with small amount of $80 \% \mathrm{v} / \mathrm{v}$ ethanol.

3. Harvest subcutaneous tumor from the mouse flank using forceps and scissors in a tissue culture hood. Cut the skin open in place of the tumor using scissors and then separate the tumor from adjacent healthy tissue using forceps and scissors.

NOTE: Depending of the purpose of the experiment perform this and following steps under aseptic conditions. In this study all steps were carried out in a cell culture hood under aseptic conditions in order to being able to cultivate the cells.

4. Prepare the tumor sample for digestions by removing fat (as this will make the sample float) and necrotic areas (as this will result in decreased viability) by cutting it away using forceps and a scalpel. Mince the tissue into pieces of $2-4 \mathrm{~mm}$ by using scalpels and forceps.

5. Transfer the tissue pieces into the $C$ Tube containing the digestion mix.

6. Close the $\mathrm{C}$ Tube and make sure it is tightly closed. Attach it upside down onto the sleeve of the benchtop tissue dissociator and make sure that the tissue pieces are located in the area of the rotor/stator. 
7. Choose the mode "h tumor_01" by pressing the "up" or "down" button until the mode appears in the display and then pressing the "start" button.

1. If using the heating function of the benchtop tissue dissociator, make sure to also attach the heater. Then run mode $37 \mathrm{C} h$ TDK 1 (for soft tumors), 37C_h_TDK_2 (for medium tumors) or 37C_h_TDK_3 (for hard tumors) by clicking the folder symbol on the touchscreen until the folder "Miltenyi" appears.

2. To choose the mode, click the up or down arrow until the dissociation mode 37C_h_TDK_2 is highlighted. The sleeves of the benchtop tissue dissociator are depicted as positions on the display of the device. Choose the positions samples are run on by clicking them on the touchscreen. Press "start" to run the mode and continue with step 2.16.

NOTE: Depending on the tumor type another dissociation program might be suitable (see step 2.7.1).

8. Observe a window showing "Termination of the program" on the screen. After termination of the program, detach $\mathrm{C}$ Tube from the benchtop tissue dissociator.

9. Incubate sample for $30 \mathrm{~min}$ at $37^{\circ} \mathrm{C}$ under continuous rotation, e.g., by using a tube rotator.

10. After incubation attach $C$ Tube upside down onto the sleeve of the benchtop tissue dissociator. Again make sure that the sample material is located in the area of the rotor/stator.

11. Run the dissociation program h_tumor_02 (for soft and medium tumors) or h_tumor_01 (for hard tumors) as described in step 2.7.1.

12. After termination of the program, detach $\mathrm{C}$ Tube from the benchtop tissue dissociator.

13. Incubate sample for $30 \mathrm{~min}$ at $37^{\circ} \mathrm{C}$ under continuous rotation.

14. Attach $C$ Tube upside down onto the sleeve of the benchtop tissue dissociator after incubation. Again make sure that the sample material is located in the area of the rotor/stator.

15. Run the dissociation program h_tumor_03 (for soft tumors), h_tumor_02 (for medium tumors) or h_tumor_01 (for hard tumors) as described in step 2.7 .

16. To collect the sample material at the tube bottom perform a short centrifugation step (at $100 \times \mathrm{g}$ for $10 \mathrm{sec}$ ).

17. Resuspend the cells and apply to a cell strainer with $70 \mu \mathrm{m}$ nesh size placed on a $50 \mathrm{ml}$ tube. Wash the cell strainer with $20 \mathrm{ml}$ of RPMl 1640 or DMEM.

18. Centrifuge cell suspension at $300 \times \mathrm{g}$ for $7 \mathrm{~min}$. Aspirate supernatant completely.

19. Resuspend cells in $5 \mathrm{ml}$ of buffer for counting. Proceed with Mouse Cell Depletion by magnetic cell separation (section 3 ).

\section{Mouse Cell Depletion by Magnetic Cell Separation Technology}

NOTE: Volumes for magnetic labeling given below are for up to $2 \times 10^{6}$ tumor cells or $10^{7}$ total cells including red blood cells. Depending on the tumor size lower or higher cell numbers will be obtained. In case of fewer cells, use the same volumes as indicated. When using higher cell numbers, scale up all reagent volumes and total volumes accordingly (e.g., for $4 \times 10^{6}$ tumor cells or $2 \times 10^{7}$ total cells, use twice the volume of all indicated reagent volumes and total volumes).

1. Determine cell number of an aliquot from cell suspension in step 2.21 using a microscope and suitable chamber for counting. NOTE: At this point the cell suspension consists of a heterogeneous mixture of human tumor cells as well as mouse stromal and blood cells, including red blood cells. The composition of the cells strongly depends on tumor type and region for transplantation.

2. When performing flow cytometric analysis after removal of mouse cells withdraw $100 \mu \mathrm{l}$ aliquots to suitable tubes for an unstained and two single color control staining's for flow cytometric analysis. Store them in a refrigerator at $2-8{ }^{\circ} \mathrm{C}$ until further staining steps (section 4).

3. Centrifuge cell suspension at $300 \mathrm{xg}$ for $10 \mathrm{~min}$. Discard the supernatant.

4. Resuspend cell pellet in $80 \mu \mathrm{l}$ of buffer per $2 \times 10^{6}$ tumor cells or $10^{7}$ total cells Add $20 \mu \mathrm{l}$ of the magnetic labeling reagent for mouse cells.

5. Mix well and incubate for $15 \mathrm{~min}$ in the refrigerator $\left(2-8^{\circ} \mathrm{C}\right)$. During magnetic labeling, prepare large scale columns (LS columns) for magnetic separation, by placing it in the magnetic field of a suitable magnet (see Table of Materials and Equipment) according to the manufacturer's protocol. Rinse the column with $3 \mathrm{ml}$ of buffer.

6. Adjust the sample volume to $500 \mu$ l using buffer for up to $2 \times 10^{6}$ tumor cells or up to $10^{7}$ total cells. (Up to $1 \times 10^{7}$ tumor cells or up to $5 \times 10^{7}$ total cells can be processed on one LS Column. When working with more cells split the sample onto multiple LS Columns).

1. When performing flow cytometric analysis, molecular analysis or comparing fractions in cultures, withdraw a $50 \mu \mathrm{l}$ aliquot as unsorted sample. Store it in a refrigerator at $2-8^{\circ} \mathrm{C}$ until further processing.

7. Apply $500 \mu \mathrm{l}$ of the cell suspension onto the previously prepared column. Collect flow-through containing unlabeled target cells, representing the enriched human tumor cells. NOTE: When working with higher cell numbers up to $2.5 \mathrm{ml}$ of cell suspension that was prepared according to step 3.5 can be applied to one column.

8. Wash column with $2 \times 1 \mathrm{ml}$ of buffer. Collect the cells that pass through and combine with the flow-through from step 3.6. Perform washing steps by adding the $1 \mathrm{ml}$ buffer aliquots as soon as the column reservoir is empty. NOTE: The flow-through contains purified human tumor cells.

9. To also collect the mouse cells retained in the column, remove column from the separator and place it on a suitable tube. Pipette $3 \mathrm{ml}$ of buffer onto the column and immediately use the plunger to flush out the mouse cells by firmly pushing it into the column.

10. Spin down the fractions and control samples at $300 \mathrm{xg}$ for $10 \mathrm{~min}$. Aspirate supernatant completely and resuspend cells in buffer, culture medium or freeze pellet in liquid nitrogen, depending on the desired downstream application.

\section{Downstream Analyses}

1. Staining for Flow Cytometric Analysis

NOTE: Before carrying out further downstream analyses, a part of cells obtained from mouse cell depletion procedure can be analyzed (e.g., assess purity and yield) in flow cytometry.

1. For flow cytometric analyses, spin at least $10 \%$ of negative and positive fraction obtained from magnetic cell separation procedure (section 3 ) as well as the control samples from step 3.2 and 3.5 at $300 \times \mathrm{g}$ for $10 \mathrm{~min}$. 
2. Discard the supernatant. Resuspend the cell pellet of the staining control samples from step 3.2 in $90 \mu$ l of buffer. Resuspend the fractions obtained from magnetic cell separation procedure in $80 \mu \mathrm{l}$ of buffer.

3. Add $10 \mu \mathrm{l}$ of mouse FcR Blocking Reagent to all samples. Mix well and incubate for $5 \mathrm{~min}$ at $2-8^{\circ} \mathrm{C}$ in a refrigerator.

4. Add $10 \mu \mathrm{l}$ of anti-human EpCAM-PE (equals antibody dilution of 1:10) and $25 \mu \mathrm{l}$ of anti-mouse-APC cocktail (equals antibody dilution of $1: 4)$ to the samples from section 3. Add $10 \mu$ of anti-human EpCAM-PE to one single color control sample (equals antibody dilution of $1: 10$ ) and $10 \mu$ l of anti-human EpCAM-APC to the other (equals antibody dilution of 1:10). Mix all samples and incubate for 10 min at 2 $-8{ }^{\circ} \mathrm{C}$ in a refrigerator.

NOTE: EpCAM is not suitable as tumor cell marker of any tumor type. For the tumors used in this study EpCAM expression was known.

5. To wash the samples, add $1 \mathrm{ml}$ of buffer each and spin at $300 \mathrm{xg}$ for $5 \mathrm{~min}$.

6. Discard the supernatant and resuspend the cell pellet to a concentration for flow cytometric analysis. To exclude dead cells add propidium iodide $(1 \mu \mathrm{g} / \mathrm{ml})$.

7. Perform flow cytometric analysis using a suitable flow cytometer according to manufacturer's protocols.

\section{Cultivation of Human Tumor Cells}

NOTE: In addition to flow cytometry, cells obtained from the separation procedure can be cultured and used for further assays in vitro.

1. Resuspend the sorted fraction from section 3 in culture medium to a concentration of $5 \times 10^{5} \mathrm{cells} / \mathrm{ml}$.

NOTE: Depending on the tumor type coating, suitable seeding density, and cultivation conditions can differ.

2. Add $500 \mu \mathrm{l}$ of cell suspension into the wells of a 24 well plate coated with $0.1 \%$ gelatin.

3. Incubate cells in a cell culture incubator at $37^{\circ} \mathrm{C}$ and $5 \%$. NOTE: Optimal culture conditions depend on the tumor type used. Therefore, apply culture conditions suitable for the tumor type used.

\section{Immunofluorescence Staining of Cultured Cells}

1. Prepare blocking solution by adding $10 \%$ FCS and $0.1 \%$ Triton X-100 to $1 \times$ PBS

2. Discard culture medium. Add $500 \mu$ of $1 x$ PBS per well to wash cells. Incubate for 2 min at room temperature (RT), then discard $1 x$ PBS completely.

3. Fix cells by adding $300 \mu \mathrm{l}$ of $4 \%$ PFA solution. Incubate at RT for $15 \mathrm{~min}$.

4. Discard PFA solution. Wash fixed cells 3 times as indicated in step 4.3.2.

5. Perform permeabilization and blocking by adding $300 \mu \mathrm{l}$ of blocking per well. Incubate at RT for $30 \mathrm{~min}$.

6. Discard blocking solution. Wash cells as indicated in step 4.3.2.

7. Add $300 \mu \mathrm{l}$ of primary antibody diluted in blocking solution to the cells. Anti-human EpCAM antibody is diluted 1:40, Vimentin antibody is diluted 1:200. Incubate overnight at $2-8^{\circ} \mathrm{C}$.

Note: The antibodies used are not suitable for any tumor type as EpCAM might not be expressed on tumor cells and/or vimentin is also expressed by the tumor cells. Make sure the antibodies used are suitable for the xenograft model.

8. Discard primary antibody solution. Wash cells 3 times as indicated in step 4.3.2.

9. Add $300 \mu \mathrm{l}$ of secondary antibody diluted in blocking solution to the cells. Both antibodies, anti-rabbit IgG-Alexe594 and anti-mouse IgG-Alexa488 are diluted 1:400. Incubate for $1 \mathrm{hr}$ in the dark at RT.

10. Discard secondary antibody solution. Wash cells 2 times as indicated in step 4.3.2.

11. Add $300 \mu \mathrm{l}$ of DAPI solution $(0.1 \mu \mathrm{g} / \mathrm{ml})$ to each well. Incubate for $10 \mathrm{~min}$ in the dark at RT.

12. Discard DAPI solution. Wash cells 3 times as indicated in step 4.3.2.

13. Add $500 \mu \mathrm{l}$ of $1 \mathrm{x}$ PBS to each well. Perform fluorescence microscopy using a suitable microscope.

\section{Whole Exome Sequencing}

1. For Whole exome sequencing (WES) freeze pellets from step 3.9 in liquid nitrogen. Store and collect samples at $-80^{\circ} \mathrm{C}$.

2. After collecting the cells, perform exome capturing and whole exome sequencing in accordance to manufacturer's instructions of the respective kit (see Table of Materials and Equipment).

\section{Representative Results}

Different approaches have been proposed to identify or deplete mouse cells from xenografted human tumors, for example a combination of mouse-specific antibodies against CD45 and MHC class I. However, only subpopulations of mouse cells were detected using these combinations whereas the proposed combination detected CD45 and MHC class I expressing mouse cells as well as the rest of the mouse cells found in target tissues for transplantation (Figure 1). Utilizing this novel combination of antibodies for magnetic cell sorting, human tumor cells could be isolated independent of tumor type (Figure 2).

Cells obtained from magnetic cell separation procedure-based mouse cell removal could be cultured, leading to pure cultures of human tumor cells (Figure 4). In addition, viable mouse cells could be obtained and cultured from the same sample. Besides removal of mouse cells, also debris was removed upon mouse cell depletion (MCD) (Figure 3).

The comprehensive depletion of mouse cells as well as debris removal lead to improved molecular downstream analyses. This was demonstrated by comparing results obtained from whole exome sequencing (WES) carried out on bulk tumor pieces and mouse cell depleted samples from three different patient-derived xenograft tumor models (Figures 5 - 9). Our data indicate that the removal of mouse cells before performing WES on xenograft tumor samples not only significantly increases the total amount of reads (Figure 5) but also considerably reduces the number of host derived reads mapped to the human reference genome (Figure 6B). As these erroneously mapped mouse reads frequently interfere with SNP calling, we observed a strong reduction of falsely predicted SNPs after MCD (Figures 7 - 8). Finally, we showed that the in silico removal of mouse-derived reads by bioinformatics methods could not fully replace the experimental procedure, as an unambiguous sequence-based assignment to the species of origin was not possible for all reads. Furthermore, the in silico procedure could not correct for the enhanced quality and concomitant higher read coverage of the in vitro depleted samples (Figure 9). 
A Mouse skin tissue

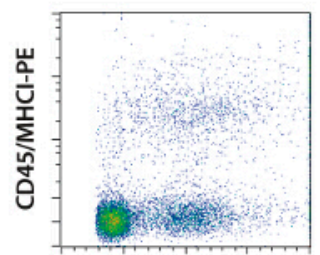

Forward scatter

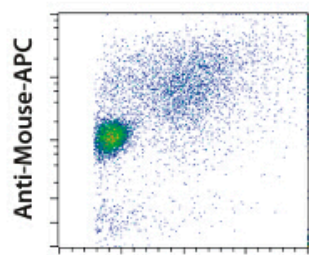

Forward scatter

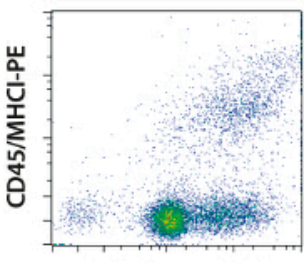

Anti-Mouse-APC

\section{B Mouse lung tissue}

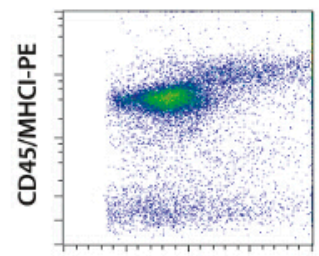

Forward scatter

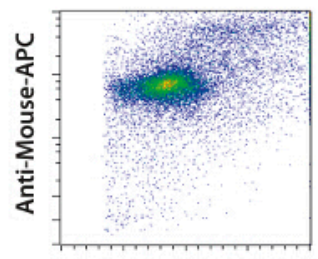

Forward scatter

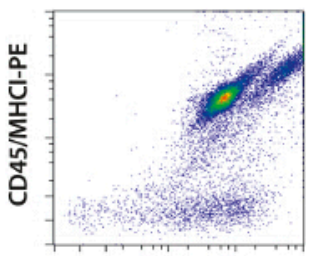

Anti-Mouse-APC

Figure 1. Establishing an Antibody Cocktail Recognizing All Mouse Cells across Multiple Organs ${ }^{14}$. Screenings in multiple organs, including skin, lung, brain, kidney and skeletal muscle, have been performed. These organs represent major target tissues for xenotransplantation. Combinations such as those recognizing mouse CD45 and MHC class I epitopes have already been used in order to deplete mouse cells after xenotransplantation. However, these marker combinations recognized only a subset of mouse cells in all analyzed tissues. In previous screenings a combination of five antibodies has been identified allowing for the comprehensive detection of all cells from mouse origin. This panel includes red blood cells and is independent of the tissue of origin (A, $\mathbf{B}$, and data not shown). Modified from ${ }^{14}$. Please click here to view a larger version of this figure. 


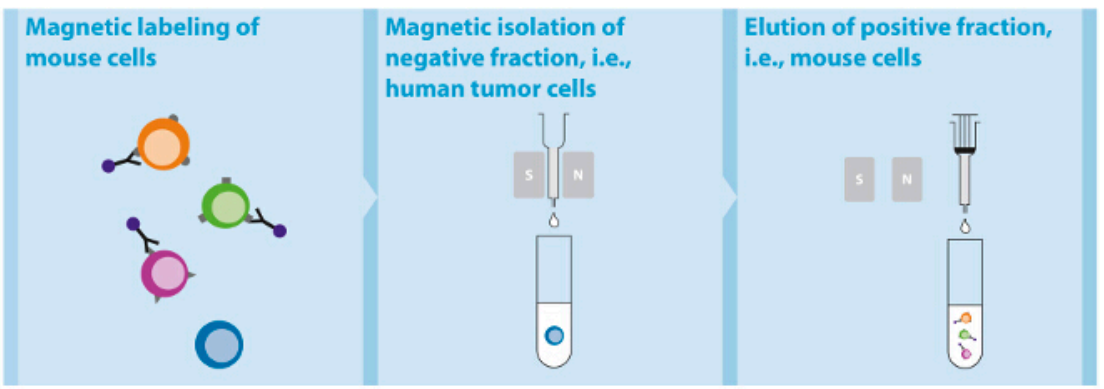

B

$$
\text { Renal cancer xenograft }
$$
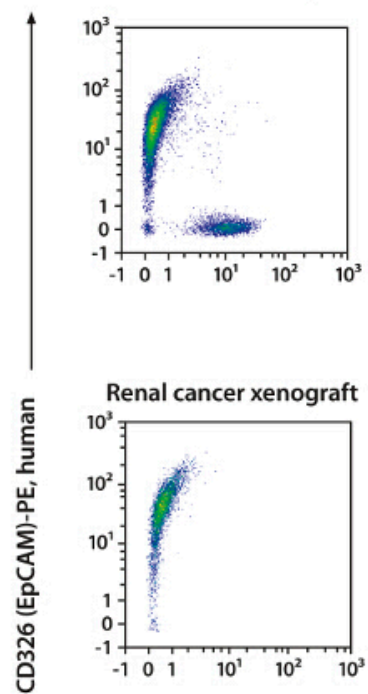

Bulk tumor Lung cancer xenograft

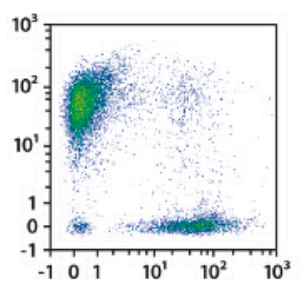

Isolated human tumor cells Lung cancer xenograft

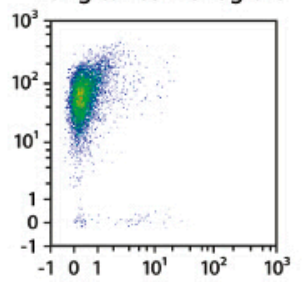

Bladder cancer xenograft

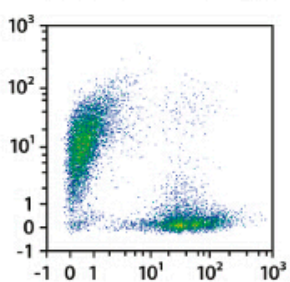

Bladder cancer xenograft

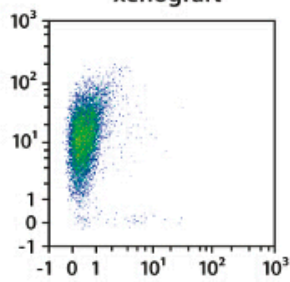

Anti-Mouse-APC

Figure 2. Reliable and Fast Depletion of Mouse Cells ${ }^{15}$. Conjugates of the antibody combination with superparamagnetic nanoparticles were used to develop an optimized protocol for depletion of mouse cells from human tumor xenografts by magnetic cell sorting (magnetic cell separation) (A). This novel protocol allowed for the elimination of $>99 \%$ of contaminating mouse cells in less than 20 min, irrespective of the tumor type. Cell fractions obtained from magnetic cell separation procedure were labeled with the pan-mouse antibody cocktail and a human specific antibody against CD326 (EpCAM) (B). Modified from ${ }^{15}$. Please click here to view a larger version of this figure. 
Original fraction

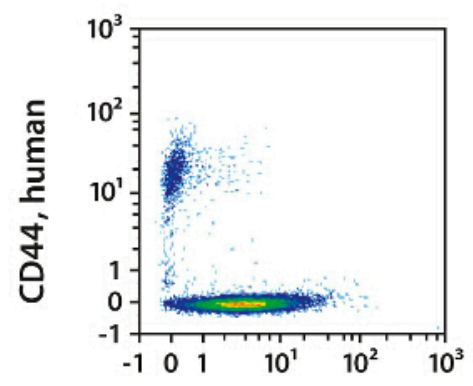

Isolated human glioblastoma cells

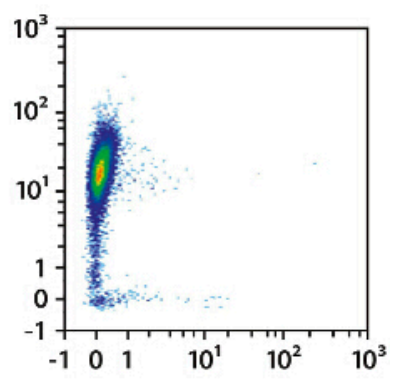

Anti-Mouse-APC

\section{Original fraction}

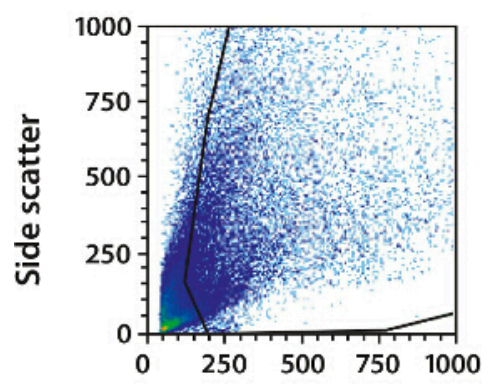

Isolated human glioblastoma cells

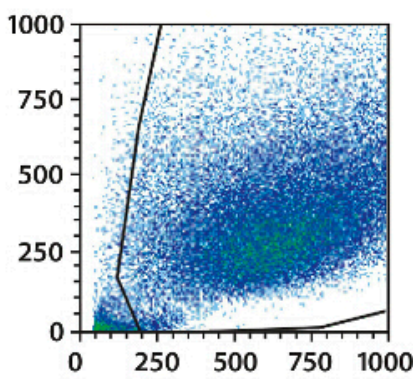

Forward scatter

Figure 3. Isolation of Human Glioblastoma Cells. Mouse Cell Depletion Kit was used to isolate human glioblastoma cells from adult mouse brain. During dissociation of neural tissue high amounts of debris is commonly generated. MCDK efficiently depletes dead cells and debris as seen by the plot showing cell size versus cell granularity. With this conjugate cocktail, it was possible to eliminate $>99 \%$ of the contaminating mouse cells and $>60 \%$ of the debris. Please click here to view a larger version of this figure. 

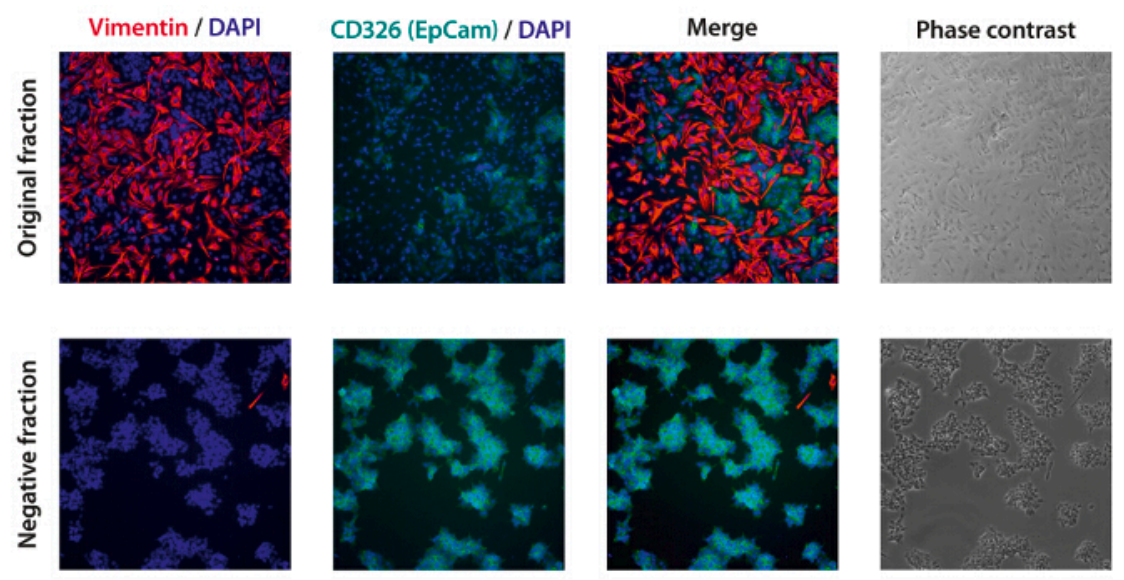

C
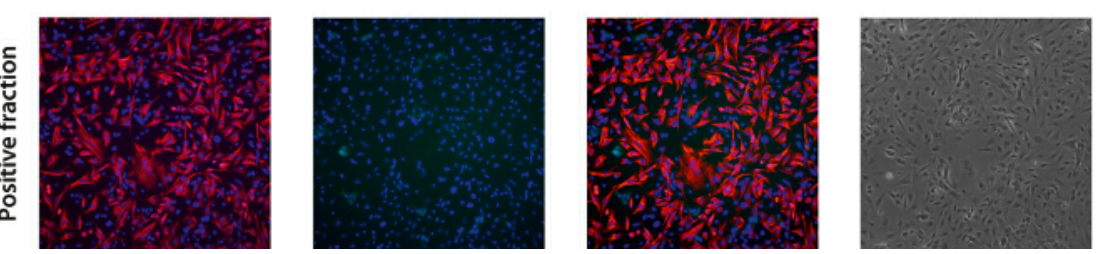

Figure 4. Depletion of Mouse Cells Facilitates Downstream Cultures of Human Tumor Cells ${ }^{14}$. Mouse fibroblasts frequently hamper the cultivation of human tumor cells after dissociation of transplanted tissues or lead to heterogeneous cultures. Fibroblasts attach and expand more efficiently, thereby overgrowing target cells. This perturbs in vitro cell culture assays (e.g., drug cytotoxicity testing), since mathematical correction for effects arising from contaminating mouse cells is impossible in most cases. The negative (B) and positive fractions (C) after mouse cell depletion were cultured for three days, fixed and stained for a mouse-specific fibroblast marker (Vimentin) and the human-specific tumor marker CD326 (EpCAM). As a control, the original fraction containing unseparated cells (A) was cultured and stained as well. Even after three days of culture, a nearly pure population of human tumor cells was observed in the negative fraction (B), whereas the unsorted fraction (A) was almost overgrown by fibroblasts. Only a minor portion of target cells was lost to the positive fraction (C). Modified from ${ }^{14}$. Please click here to view a larger version of this figure. 


\begin{tabular}{llll}
\hline Xenograft & Description & Cluster density $\left(\mathrm{K} / \mathrm{mm}^{2}\right)$ & Estimated yield (MB) \\
\hline Kidney & Bulk tumor & 777 & 2841.8 \\
\hline Kidney & Isolated human tumor cells & 1179 & 4195.8 \\
Lung & Bulk tumor & 793 & 2904.4 \\
Lung & Isolated human tumor cells & 1020 & 3678 \\
Bladder & Bulk tumor & 843 & 3063.6 \\
Bladder & Isolated human tumor cells & 1075 & 3859.2
\end{tabular}

B

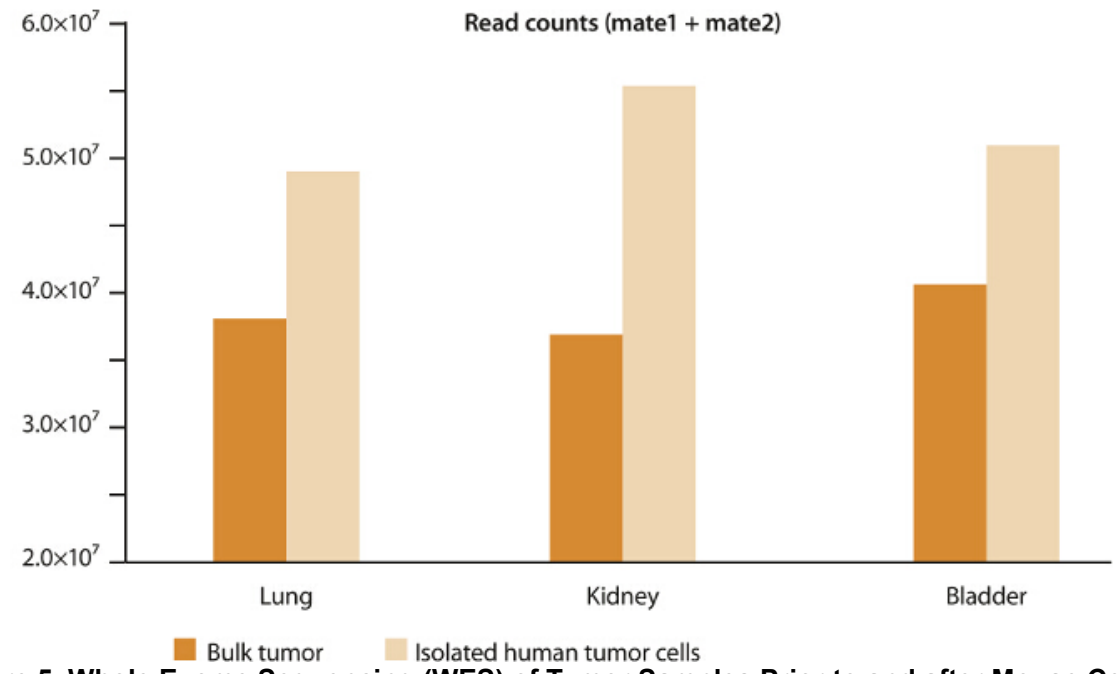

Figure 5. Whole Exome Sequencing (WES) of Tumor Samples Prior to and after Mouse Cell Depletion (MCD) ${ }^{15}$. We conducted WES on three different xenograft models derived from human kidney, lung and bladder cancer in order to assess the impact of MCD on the quality of next-generation sequencing data. DNA from bulk tumor and from isolated tumor cells after mouse cell depletion was used to generate exomecaptured sequencing libraries applying an exome capture kit. For sequencing on a desktop sequencer instrument, a desktop sequencer reagent kit 150 cycles, was utilized to generate 75 -bp paired-end reads. A significant increase $(p<0.05)$ in cluster density $(\mathbf{A})$ as well as an average increase in read counts of $33 \%$ (B) was observed for the samples depleted of mouse cells, indicating improved sample quality. Correspondingly, a strong reduction of debris and dead cells upon MCD could also be demonstrated by flow cytometry analysis (see Figure 3 ). Modified from ${ }^{15}$. Please click here to view a larger version of this figure. 

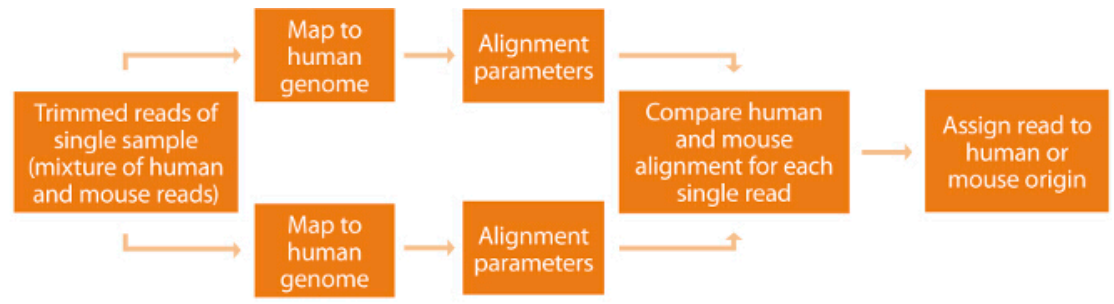

B

Bulk tumor

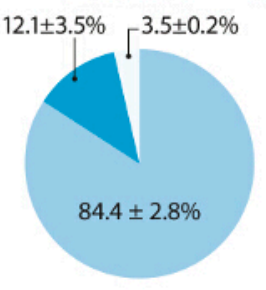

origin: human

origin: mouse

no decision possible

origin: mouse, not mapped to human

origin: mouse, (erroneously) mapped to human

neither mapped to human or mouse

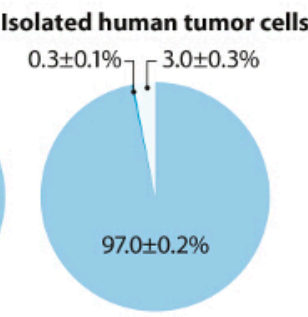

Bulk tumor

(bladder)

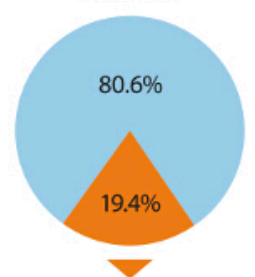

$19.4 \%$ total:

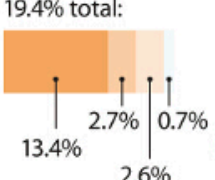

Isolated human

tumor cells (bladder)

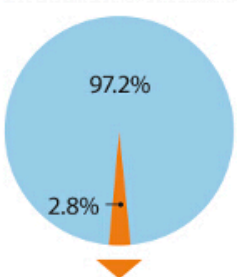

$2,8 \%$ total:

Figure 6. Mouse Cell Depletion (MCD) Strongly Reduces Number of Erroneously Mapped Mouse Reads after Whole Exome Sequencing

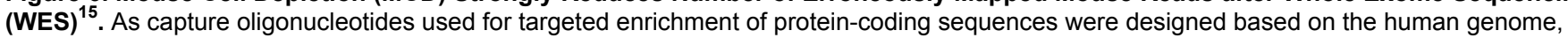
an initial pre-enrichment of DNA fragments of human origin from the mixture of mouse and human cells was expected. In order to assess the number of capture oligonucleotides that might cross-hybridize with mouse genomic DNA, we conducted BLAST searches of each single rapid capture exome probe against mouse genome. The resulting alignment parameters were used to determine possible cross-hybridization. Depending on the selection thresholds (alignment length, no. of mismatches, no. of gaps), we predicted a cross-reactivity of $5-10 \%$ of capture probes with mouse transcripts (data not shown). After adapter clipping (trimmomatic $v 0.32^{16}$ ), we mapped the reads of all samples against human and mouse genomes (bwa v0.7.12 ${ }^{17}$ ) and determined their putative origin based on the respective alignment parameters (LINUX shell, command-line Perl) (A). An average of $12 \%$ of reads derived from bulk tumor samples was attributed to mouse cells. This amount could be reduced to $0.3 \%$ by prior depletion of mouse cells (B). As on average $15 \%$ of the mouse-derived reads mapped erroneously to the human genome, corresponding to $1.9 \%$ of total reads in the bulk tumor samples and $0.04 \%$ in the isolated tumor cells, a strong positive influence of mouse cell depletion on downstream analyses can be expected. Figure 6B exemplifies the detailed read assignment for bulk tumor and isolated human tumor cells derived from the bladder cancer xenograft. Modified from ${ }^{15}$. Please click here to view a larger version of this figure. 
A

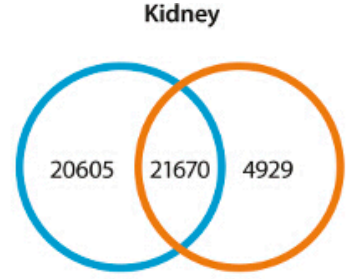

$48.7 \%$

$18.5 \%$

B

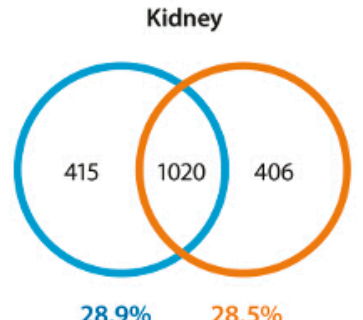

$28.9 \%$

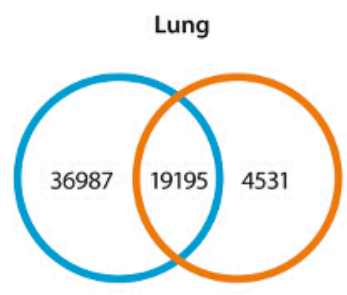

$65.8 \%$

$19.1 \%$
Lung

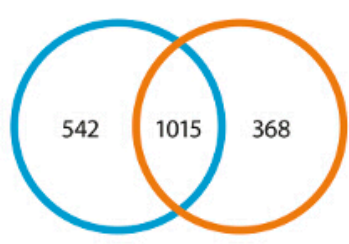

$34.8 \%$

$26.6 \%$

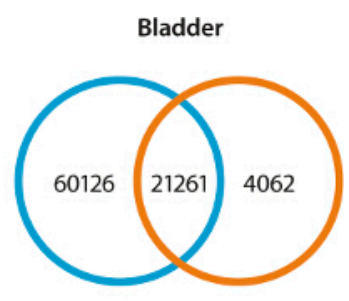

$73.9 \% \quad 16.0 \%$

Bladder

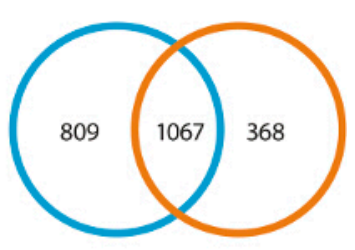

$43.1 \% \quad 25.6 \%$

non-depleted $\square$ MCD

Figure 7. MCD Strongly Reduces the Number of Falsely Predicted SNPs ${ }^{15}$. In order to determine the impact of mouse reads mapped to the human genome, we determined the number of predicted SNPs for the xenograft samples prior to and after MCD. As no healthy tissue was available for comparison, a SNP was defined as a difference between the sequenced sample and the reference genome (hg19). After removal of duplicate reads, SNP and INDEL calling was conducted ${ }^{18}$ and was restricted to the regions targeted by an exome capture kit. $63 \pm 10 \%$ of all SNPs predicted for the bulk tumor samples were no longer detected after mouse cell depletion, $18 \pm 1 \%$ were specific for the isolated human tumor cells (A). While the former were mainly caused by erroneously mapped mouse reads the latter seemed to be detected due to higher read counts and accordingly higher coverage within the isolated human tumor cell samples. This effect was also visible for predicted INDELs (B). Modified from ${ }^{15}$. Please click here to view a larger version of this figure. 


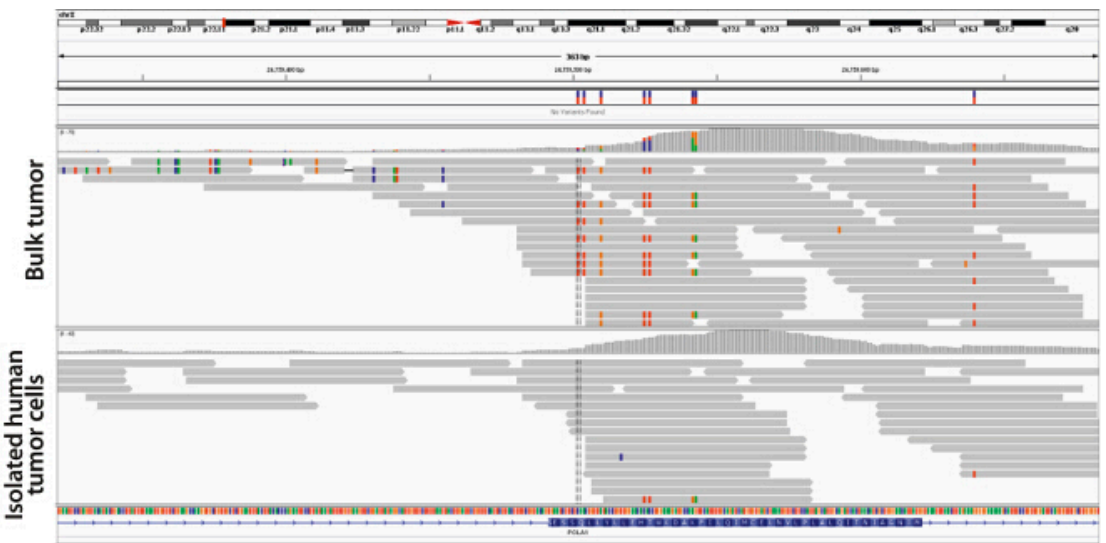

B
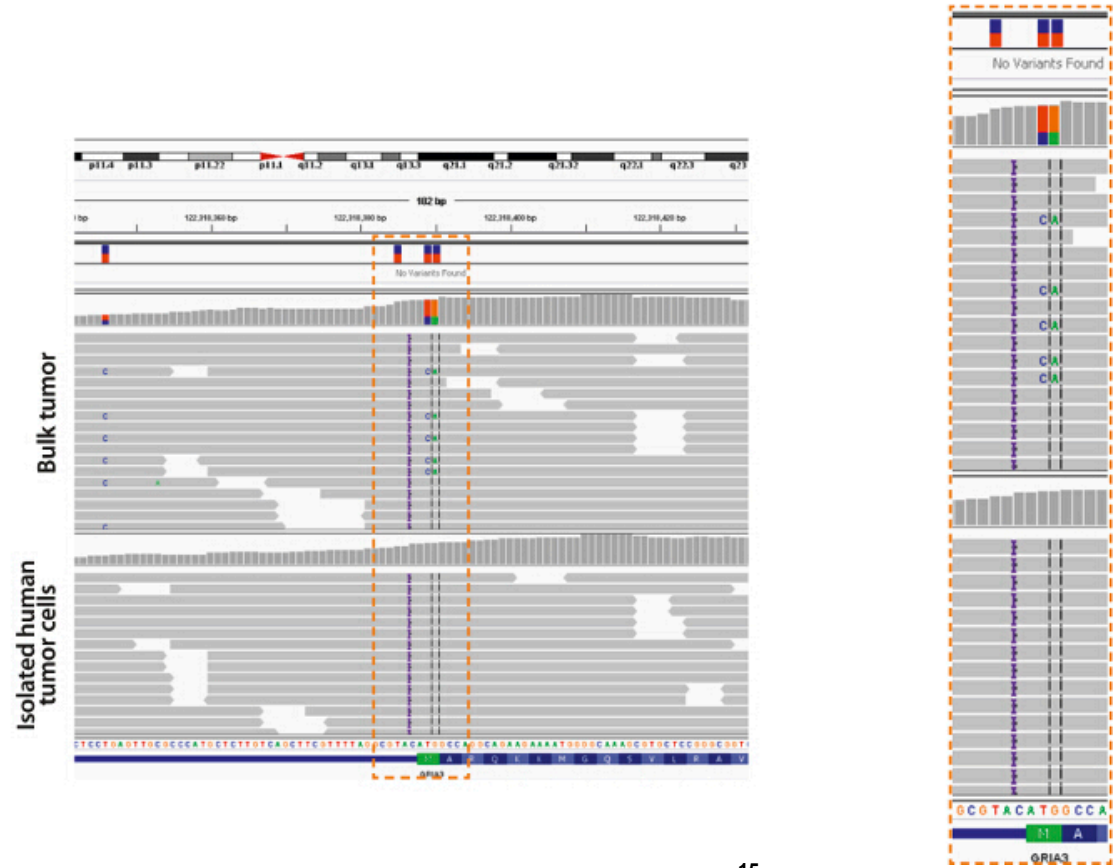

Figure 8. MCD Improves the Prediction of High-impact SNPs ${ }^{15}$. (A) Impact of MCD on SNP prediction within a protein-coding exon of the POLA1 gene ${ }^{19}$ is exemplified. While erroneously mapped mouse reads caused a number of falsely predicted SNPs in the bulk kidney cancer xenograft, these SNPs were missing after MCD. In addition, MCD also improved the prediction of high-impact SNPs. For example, mouse reads mapped to the human reference genome in the bulk tumor sample resulted in the wrongly predicted destruction of the start codon of the GRIA3 gene (B). Modified from ${ }^{15}$. Please click here to view a larger version of this figure.

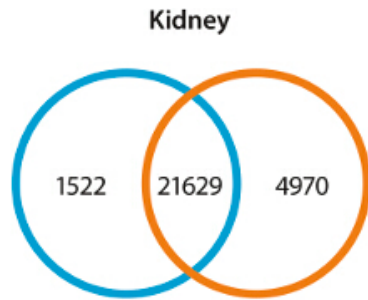

$6.5 \%$

$18.7 \%$

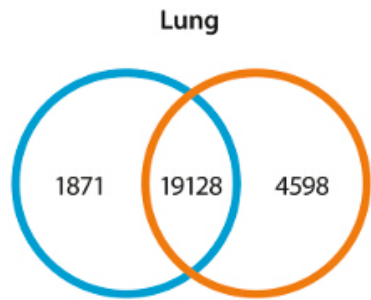

$8.9 \%$

$19.4 \%$

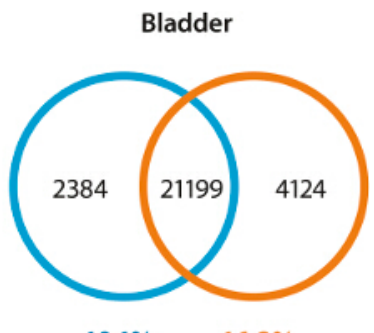

$10.1 \%$

$16.3 \%$

In silico In vitro

Figure 9. In Silico Depletion of Mouse-derived Reads Cannot Fully Replace In Vitro MCD ${ }^{15}$. The reads predicted to be of mouse origin were computationally removed from the sequencing data, and SNP calling was repeated. By this approach, the number of SNPs predicted for the bulk tumor samples was considerably reduced from $63 \%$ to $8.5 \%$ of the total number of SNPs (compare with Figure 8). However, this in silico approach could not completely replace in vitro MCD, especially if the improved sample quality and the concomitant increase in read counts and coverage are considered. Modified from ${ }^{15}$. Please click here to view a larger version of this figure. 


\section{Discussion}

We have developed a fast and easy method to isolate untouched human tumor cells from xenografted tumor tissue. This procedure is based on the comprehensive depletion of cells of mouse origin by combining automated tissue dissociation and magnetic cell sorting. Besides depletion of all mouse cells also the amount of dead cells and debris is significantly reduced in the target cell fraction. Taken together, this method significantly improves molecular downstream analyses and cultivation of human tumor cells.

In contrast to alternative methods, there is no need for knowledge of tumor cell specific markers, adjustment to varying compositions of mouse cells or establishment of algorithms. The presented method is independent of the mouse strain and tumor type. Therefore, a bias through isolation of human tumor subpopulations on the basis of single human specific markers which may vary in expression, as shown for EpCAM ${ }^{11}$, is avoided. Furthermore, it is not limited to tumor material but can be used for any kind of xenotransplanted tissue as mouse cells are targeted instead of specific human tumor subpopulations (data not shown).

Comprehensive removal of mouse cells is facilitated by targeting surface epitopes exclusively expressed on cells of mouse origin. Apart from the well-established method for tumor dissociation as presented in this study, there are many procedures using different kinds of enzymes. Preservation of cell surface epitopes is a prerequisite for this novel method, but also for accurate analyses of human tumor cell populations. Therefore, it is crucial that gentle enzymes are used for digestion of tumor tissue. Thus, mouse cell depletion can only be used in combination with enzymatic digestion procedures that evidently do not affect epitopes targeted during mouse cell depletion procedure. In case of doubt this can only be verified by the respective manufacturer.

This method also works for human circulating tumor cells (CTCs). However, as frequencies of target cells are usually $<0.1 \%$ removal of mouse cells additionally requires red blood cell lysis and purities of more than $50 \%$ cannot be expected. In this case, mouse cell depletion can be used as pre-enrichment of CTCs followed by an enrichment using positive markers (data not shown).

Removal of mouse cells significantly improves the culture of human tumor cells by avoiding culture overgrow of mouse fibroblasts. In addition, the analysis of human tumor xenografts by next-generation sequencing is significantly improved and standardized. As this effect was observed although a targeted human sequence-specific selection has been carried out during exome enrichment, the influence on whole genome and whole transcriptome sequencing are expected to be even more prominent.

Moreover, the presented method facilitates accurate molecular studies of tumor subpopulations within xenotransplanted human tumors. Removal of mouse cells from a respective sample in the first step and subsequently sorting human tumor cells in two different subpopulations enables direct molecular comparison of the tumor subpopulation of interest to human bulk tumor cells ${ }^{20}$. Differential gene expression between tumor cell subtypes can be more reliably assessed as it is not affected by cross-hybridization of mouse-derived molecules.

\section{Disclosures}

David Agorku, Stefan Tomiuk, Stefan Wild, Silvia Rüberg, Lisa Zatrieb, Andreas Bosio and Olaf Hardt are or were employees of Miltenyi Biotec $\mathrm{GmbH}$, Germany. Kerstin Klingner and Julia Schüler are employees of Oncotest GmbH, Germany.

\section{Acknowledgements}

We are grateful to Janina Kuhl, Nadine Chelius, Petra Kussmann, Lena Willnow and Dorothee Lenhard for excellent technical assistance.

\section{References}

1. Daniel, V. C. et al. A primary xenograft model of small-cell lung cancer reveals irreversible changes in gene expression imposed by culture in vitro. Cancer Res. 69 (8), 3364-3373 (2009).

2. Tentler, J. J. et al. Patient-derived tumour xenografts as models for oncology drug development. Nat Rev Clin Oncol. 9 (6), $338-350$ (2012).

3. DeRose, Y. S. et al. Tumor grafts derived from women with breast cancer authentically reflect tumor pathology, growth, metastasis and disease outcomes. Nat Med. 17 (11), 1514-1520 (2011).

4. Siolas, D., \& Hannon, G. J. Patient-derived tumor xenografts: transforming clinical samples into mouse models. Cancer Res. 73 (17), 5315-5319 (2013).

5. Wong, S. Q. et al. Targeted-capture massively-parallel sequencing enables robust detection of clinically informative mutations from formalinfixed tumours. Sci Rep. 33494 (2013).

6. Sausville, E. A., \& Burger, A. M. Contributions of human tumor xenografts to anticancer drug development. Cancer Res. 66 (7), $3351-3354$, discussion 3354 (2006).

7. Rubio-Viqueira, B., \& Hidalgo, M. Direct in vivo xenograft tumor model for predicting chemotherapeutic drug response in cancer patients. Clin Pharmacol Ther. 85 (2), 217-221 (2009).

8. Reubinoff, B. E., Pera, M. F., Fong, C. Y., Trounson, A., \& Bongso, A. Embryonic stem cell lines from human blastocysts: somatic differentiation in vitro. Nat Biotechnol. 18 (4), 399-404 (2000).

9. Merk, J., Rolff, J., Becker, M., Leschber, G., \& Fichtner, I. Patient-derived xenografts of non-small-cell lung cancer: a pre-clinical model to evaluate adjuvant chemotherapy? Eur J Cardiothorac Surg. 36 (3), 454-459 (2009).

10. Baiocchi, M., Biffoni, M., Ricci-Vitiani, L., Pilozzi, E., \& De Maria, R. New models for cancer research: human cancer stem cell xenografts. Curr Opin Pharmacol. 10 (4), 380-384 (2010).

11. Gires, O., \& Stoecklein, N. H. Dynamic EpCAM expression on circulating and disseminating tumor cells: causes and consequences. Cell Mol Life Sci. 71 (22), 4393-4402 (2014). 
12. Zhang, C. C. et al. Synergistic effect of the gamma-secretase inhibitor PF-03084014 and docetaxel in breast cancer models. Stem Cells Transl Med. 2 (3), 233-242 (2013).

13. Boven, E. et al. Phase II preclinical drug screening in human tumor xenografts: a first European multicenter collaborative study. Cancer Res. 52 (21), 5940-5947 (1992).

14. Agorku, D., Hardt, O., \& Bosio, A. Depletion of mouse cells from human tumor xenografts significantly reduces bias in molecular analysis and improves culture of target cells. Abstract 95. AACR. (2014).

15. Agorku, D. et al. Next-generation sequencing of human tumor xenografts is significantly improved by prior depletion of mouse cells. Abstract 1455. AACR. (2015).

16. Bolger, A. M., Lohse, M., \& Usadel, B. Trimmomatic: a flexible trimmer for Illumina sequence data. Bioinformatics. 30 (15), $2114-2120$ (2014)

17. Li, H., \& Durbin, R. Fast and accurate short read alignment with Burrows-Wheeler transform. Bioinformatics. 25 (14), $1754-1760$ (2009).

18. Koboldt, D. C., Larson, D. E., \& Wilson, R. K. Using VarScan 2 for Germline Variant Calling and Somatic Mutation Detection. Curr Protoc Bioinformatics. 441514 11-15 1417 (2013).

19. Robinson, J. T. et al. Integrative genomics viewer. Nat Biotechnol. 29 (1), 24-26 (2011).

20. Aloia, A. et al. The sialyl-glycolipid stage-specific embryonic antigen 4 marks a subpopulation of chemotherapy-resistant breast cancer cells with mesenchymal features. Breast Cancer Res. 17 (1), 146 (2015). 